\title{
Label Adherence for Non-Vitamin K Antagonist Oral Anticoagulants in a Prospective Cohort of Asian Patients with Atrial Fibrillation
}

\author{
So-Ryoung Lee ${ }^{1 *}$, Young Soo Lee ${ }^{2 *}$, Ji-Suck Park ${ }^{1}$, Myung-Jin Cha ${ }^{1}$, Tae-Hoon Kim³ ${ }^{3}$ Junbeom Park ${ }^{4}$, \\ Jin-Kyu Park ${ }^{5}$, Jung-Myung Lee ${ }^{6}$, Ki-Woon Kang ${ }^{7}$, Jaemin Shim ${ }^{8}$, Jae-Sun Uhm ${ }^{3}$, Jun Kim ${ }^{9}$, Changsoo Kim ${ }^{10}$, \\ Jin-Bae Kim ${ }^{6}$, Hyung Wook Park ${ }^{11}$, Boyoung Joung ${ }^{3}$, and Eue-Keun Choi ${ }^{1}$ \\ ${ }^{1}$ Department of Internal Medicine, Seoul National University Hospital, Seoul; \\ ${ }^{2}$ Division of Cardiology, Catholic University of Daegu, Daegu; \\ ${ }^{3}$ Division of Cardiology, Yonsei University College of Medicine, Seoul; \\ ${ }^{4}$ Department of Cardiology, College of Medicine, Ewha Womans University, Seoul; \\ ${ }^{5}$ Division of Cardiology, Hanyang University Medical Center, Seoul; \\ ${ }^{6}$ Division of Cardiology, Kyung Hee University Medical College, Seoul; \\ ${ }^{7}$ Division of Cardiology, Eulji University Hospital, Daejeon; \\ ${ }^{8}$ Division of Cardiology, Korea University Anam Hospital, Seoul; \\ ${ }^{9}$ Department of Internal Medicine, University of Ulsan College of Medicine, Seoul; \\ ${ }^{10}$ Department of Preventive Medicine, Yonsei University College of Medicine, Seoul; \\ ${ }^{11}$ Department of Cardiovascular Medicine, Chonnam National University Medical School, Gwangju, Korea.
}

Purpose: Label adherence for non-vitamin K antagonist oral anticoagulants (NOACs) has not been well evaluated in Asian patients with non-valvular atrial fibrillation (AF). The present study aimed to assess label adherence for NOACs in a Korean AF population and to determine risk factors of off-label prescriptions of NOACs.

Materials and Methods: In this COmparison study of Drugs for symptom control and complication prEvention of AF (CODE-AF) registry, patients with AF who were prescribed NOACs between June 2016 and May 2017 were included. Four NOAC doses were categorized as on- or off-label use according to Korea Food and Drug Regulations.

Results: We evaluated 3080 AF patients treated with NOACs (dabigatran 27.2\%, rivaroxaban 23.9\%, apixaban 36.9\%, and edoxaban $12.0 \%$ ). The mean age was $70.5 \pm 9.2$ years; $56.0 \%$ were men; and the mean $\mathrm{CHA}_{2} \mathrm{DS}_{2}$-VASc score was 3.3 \pm 1.4 . Only one-third of the patients $(32.7 \%)$ was prescribed a standard dose of NOAC. More than one-third of the study population (n=1122, 36.4\%) was prescribed an off-label reduced dose of NOAC. Compared to those with an on-label standard dosing, patients with an off-label reduced dose of NOAC were older ( $\geq 75$ years), women, and had a lower body weight $(\leq 60 \mathrm{~kg})$, renal dysfunction (creatinine clearance $\leq 50 \mathrm{~mL} / \mathrm{min}$ ), previous stroke, previous bleeding, hypertension, concomitant dronedarone use, and anti-platelet use. Conclusion: In real-world practice, more than one-third of patients with NOAC prescriptions received an off-label reduced dose, which could result in an increased risk of stroke. Considering the high risk of stroke in these patients, on-label use of NOAC is recommended.

Key Words: Atrial fibrillation, non-vitamin K antagonist oral anticoagulant, drug labeling, dose

Received: October 1, 2018 Revised: January 7, 2019 Accepted: January 28, 2019

Co-corresponding authors: Eue-Keun Choi, MD, PhD, Department of Internal Medicine, Seoul National University Hospital, 101 Daehak-ro, Jongno-gu, Seoul 03080, Korea. Tel: 82-2-2072-0688, Fax: 82-2-762-9662, E-mail: choiek17@snu.ac.kr and

Boyoung Joung, MD, PhD, Division of Cardiology, Yonsei Cardiovascular Hospital, Yonsei University College of Medicine, 50-1 Yonsei-ro, Seodaemun-gu, Seoul 03722, Korea. Tel: 82-2-2228-8460, Fax: 82-2-393-2041, E-mail: cby6908@yuhs.ac

${ }^{*}$ So-Ryoung Lee and Young Soo Lee contributed equally to this work.

-The authors have no potential conflicts of interest to disclose.

(C) Copyright: Yonsei University College of Medicine 2019

This is an Open Access article distributed under the terms of the Creative Commons Attribution Non-Commercial License (https://creativecommons.org/licenses/by-nc/4.0) which permits unrestricted non-commercial use, distribution, and reproduction in any medium, provided the original work is properly cited. 


\section{INTRODUCTION}

Adjusted doses of warfarin can be prescribed according to prothrombin time in order to achieve a target therapeutic window, which necessitates frequent monitoring. In comparison, non-vitamin K antagonist oral anticoagulants (NOACs) exert a predictable effect without requiring standard anticoagulation monitoring and are suitable alternatives to warfarin for stroke prevention in patients with atrial fibrillation (AF). Their use in clinical practice is increasing rapidly..$^{2-4}$ Although the dosage of NOAC is not determined by the results of anticoagulation test results, each NOAC has a recommended dose based on clinical characteristics (e.g., age, body weight, renal function, and concomitant medications). The efficacy and safety of the recommended doses of each NOAC to prevent stroke in patients with $\mathrm{AF}$ have been confirmed in pivotal randomized clinical trials. ${ }^{5-9}$ All of these phase 3 trials, except for the RE-LY trial, have provided criteria for dose reduction, which physicians can apply to reduce NOAC doses to decrease the risk of bleeding. In real-world practice, off-label use of a reduced dose of NOAC is not uncommon. In recent reports in the U.S., $13-16 \%$ of patients received off-label NOAC doses, and the majority of these patients were prescribed with underdosing. ${ }^{10,11}$ Although reduced doses of NOACs might be considered as an improvement in safety by lowering the risk of bleeding in fragile patients, off-label underdosing of NOACs has been shown to be associated with an increased risk of stroke without a benefit in safety. ${ }^{10,11}$

Compared to Western populations, Asian patients with $\mathrm{AF}$ are smaller and have a higher risk of bleeding. Accordingly, physicians prefer to prescribe a reduced dose of NOAC more frequently than physicians in the Western countries. ${ }^{12-14}$ However, there is a paucity of information on whether these reduced doses of NOAC meet the criteria for dose reduction. Therefore, we sought to assess label adherence for NOACs and to identify risk factors associated with off-label prescriptions of NOACs in real-world practice in a Korean population.

\section{MATERIALS AND METHODS}

\section{Data source}

The COmparison study of Drugs for symptom control and complication prEvention of $\mathrm{AF}$ (CODE-AF) registry is a prospective, multicenter, observational study of AF patients enrolled at 10 tertiary centers in Korea. The study design and centers have been described previously. ${ }^{15}$ The first database of the CODE-AF registry for analysis was released in May 2017 with patients from June 2016 to April 2017. The data entered at each center were regularly audited, and the database used in this study completed data cleansing. The collected data were registered in the web-based clinical research management system iCReaT (Internet based Clinical Research and Trial management system, http://icreat.nih.go.kr) provided by the Korean government. The study protocol conforms to the ethical guidelines of the 1975 Declaration of Helsinki; the study was approved by the ethics committee of each center; and all study patients provided informed consent and were registered at ClinicalTrials.gov (NCT02786095). The study was approved by the Seoul National University Hospital Institutional Review Board (IRB Number: 1605-082-76).

\section{Study population}

Among the 6275 patients enrolled in the CODE-AF registry, we included patients who were prescribed a NOAC for stroke prevention ( $\mathrm{n}=3213)$. Patients without available dose information were excluded. Patients without information for evaluation of compliance with labeled dosing, such as age, sex, body weight, or serum creatinine value, were also excluded. We also excluded patients with end-stage renal disease who were on dialysis.

\section{Dose reduction criteria}

A standard dose was defined, according to each NOAC, as dabigatran $150 \mathrm{mg}$ twice daily, rivaroxaban $20 \mathrm{mg}$ once daily, apixaban $5 \mathrm{mg}$ twice daily, and edoxaban $60 \mathrm{mg}$ once daily. Approved dose reduction criteria were specific to each NOAC according to the following patient characteristics: baseline age, body weight, and serum creatinine level at enrollment. Creatinine clearance $(\mathrm{CrCl})$ was calculated using the Cockcroft-Gault equation.

In the present study, adherence with labeled dosing for each NOAC in each study patient was evaluated based on the Ministry of Food and Drug Safety labeling (Supplementary Table 1, only online).

\section{Evaluation of NOAC dosing according to label}

Patients were categorized into four groups based on NOAC dose and dose recommendation adherence: on-label standard dose, on-label reduced dose, underdosed, and overdosed. To identify predictors of those who had an underdosed prescription, we compared the clinical characteristics between patients who were prescribed an on-label standard dose and those with an underdose who needed to be prescribed the standard dose.

Exclusively for dabigatran, both the $150 \mathrm{mg}$ and $110 \mathrm{mg}$ twice daily doses were regarded as on-label use, because dabigatran does not have mandatory dose reduction criteria for selected patients. ${ }^{5,9}$ Therefore, dabigatran $110 \mathrm{mg}$ twice daily prescription was defined as the on-label reduced dose, regardless of patients' baseline characteristics. Instead, the Ministry of Food and Drug Safety labeling recommended the use of dabigatran $110 \mathrm{mg}$ bid in those with $\mathrm{CrCl} 30-50 \mathrm{~mL} / \mathrm{min}$ or age $\geq 75$ years. Therefore, for patients prescribed with a reduced dose of dabigatran, we defined the patients who met one of these criteria as "recommendation-concordant prescription," and those who did not meet any of these criteria as "recommendation- 
discordant prescription." ${ }^{16}$

\section{Comparison of dose reduction criteria among NOACs} Differences in dose reduction criteria for each NOAC could result in a discrepancy in prescriptions with standard or reduced doses among the NOACs. For example, one NOAC should be prescribed with a standard dose, whereas another NOAC could be prescribed with a reduced dose. To evaluate this discrepancy in dose reductions among NOACs, we applied dose reduction criteria to each NOAC of the total population, evaluated the dose reduction criteria of the four NOACs in each patient, and determined which NOAC could be prescribed as a labeled reduced dose, whereas other NOACs could not.

\section{Statistical analysis}

Continuous variables are presented as means and standard deviations. Comparison of continuous variables was performed using an independent t-test or, in case of a non-normal distribution, the Mann-Whitney test. Categorical variables are presented as numbers and percentages and were compared using the chi-square test of Fisher's exact test. To assess factors associated with the prescription of off-label reduced doses of NOACs, univariate binary logistic regression analysis was used. All variables with $p<0.1$ in the univariate analysis were used for multivariate logistic regression analysis. The $\mathrm{CHA}_{2} \mathrm{DS}_{2}$-VASc and HAS-BLED scores were excluded in the multivariable logistic regression because of interactions with variables included in the clinical scores. Sensitivity analysis was performed including $\mathrm{CHA}_{2} \mathrm{DS}_{2}$-VASc and HAS-BLED scores. Odds ratios were calculated as an estimate of the risk associated with a particular variable with a $95 \%$ confidence interval based on binomial distributions. All statistical analyses were performed with SPSS statistical package version 19.0 (IBM Corp., Armonk, NY, USA). All two-tailed $p$ values $<0.05$ were considered statistically significant.

\section{RESULTS}

\section{Baseline characteristics of the study population}

We evaluated $3080 \mathrm{AF}$ patients treated with NOACs (dabigatran $27.2 \%$, rivaroxaban $23.9 \%$, apixaban $36.9 \%$, and edoxaban $12.0 \%)$. The baseline characteristics are presented in Supplementary Table 2 (only online). The mean age was 70.5 \pm 9.2 years; $56.0 \%$ were men; $34 \%$ were under $60 \mathrm{~kg}$; and the mean $\mathrm{CHA}_{2} \mathrm{DS}_{2}$-VASc score was $3.3 \pm 1.4\left(\mathrm{CHA}_{2} \mathrm{DS}_{2}\right.$-VASc score $\geq 2$, 93.1\%). Six hundred seventy-seven (22\%) patients had moderate renal dysfunction $(\mathrm{CrCl}<50 \mathrm{~mL} / \mathrm{min})$. Eighty-eight (2.9\%) patients were prescribed dronedarone concomitantly. Among the NOAC groups, the apixaban group was older and had a higher $\mathrm{CHA}_{2} \mathrm{DS}_{2}$-VASc score than the other NOAC groups.

In the total study population, $32.7 \%$ of patients were prescribed a standard dose, and $67.3 \%$ of patients received a re- duced dose. There was a difference in the prescription patterns of dose reduction by each NOAC $(71.8 \%$ in dabigatran, $69.3 \%$ in rivaroxaban, $66.3 \%$ in apixaban and $56.6 \%$ in edoxaban). Patients taking dabigatran and rivaroxaban were prescribed a reduced dose more frequently than those taking apixaban and edoxaban, whereas edoxaban was prescribed as a reduced dose in only half of patients (all, $p<0.05$, Supplementary Table 2, only online).

\section{Label adherence for NOAC prescription}

Among the entire study population, on-label users accounted for $61.9 \%$ (31.1\% with a standard dose and $30.8 \%$ with a reduced dose). Those who were off-label underdosed comprised $36.4 \%$, and off-label overdosed included 1.6\% (Table 1, Fig. 1). Most patients taking dabigatran were prescribed labeled used, although there were a few patients with off-label use (once daily prescription or overdose prescription in 16 cases). Edoxaban showed the highest dose recommendation adherence (on-labeled use, $68 \%$ ), whereas rivaroxaban showed the lowest dose recommendation adherence (on-labeled use, 43.9\%). Among those with a standard dose ( $\mathrm{n}=1009)$, most patients $(95 \%)$ adhered with the labeling (Fig. 2). Most patients with a standard dose of dabigatran, rivaroxaban, and apixaban followed on-label prescription, while edoxaban showed a higher proportion of overdosed prescriptions, compared to other NOACs $(0,7,0$ and $20 \%$, respectively, $p<0.001$ ) (Fig. 2). The reasons for this overdose prescription of edoxaban included patients being underweight ( $\leq 60 \mathrm{~kg}, \mathrm{n}=18$ ), moderate-to-severe renal dysfunction ( $\mathrm{CrCl} 15-50 \mathrm{~mL} / \mathrm{min}, \mathrm{n}=6)$, or both ( $\mathrm{n}=8)$. Among those taking a reduced dose $(\mathrm{n}=2071)$, only $46.3 \%$ of patients had onlabel use, whereas more than half of patients (53.7\%) had offlabel use.

The proportions of off-label underdosed prescription differed among NOACs. Rivaroxaban and apixaban showed similar, but higher, prescriptions of off-label reduced doses $(78 \%$ and $83 \%$, respectively, $p=0.051$ ) than edoxaban, whereas the edoxaban group showed only $41 \%$ of off-label reduced dose prescription (rivaroxaban vs. edoxaban, apixaban vs. edoxaban, both $p<0.001)$. The reasons for off-label use in each NOAC were as follows: Among 396 patients taking underdosed rivaroxaban, 320 (81\%) patients had normal renal function, and 76 (19\%) patients were prescribed a dose less than $10 \mathrm{mg}$. Among 624 patients with underdosed apixaban, 300 (48\%) patients met one dose reduction criterion; $274(44 \%)$ patients met no dose reduction criteria; and $50(8 \%)$ patients were prescribed a once-daily dosage or less than $2.5 \mathrm{mg}$ bid. Among 87 patients with underdosed edoxaban, $79(91 \%)$ patients met none of the dose reduction criteria, and 8 patients were prescribed a $15 \mathrm{mg}$ dosage. Among the patients with a reduced dose of dabigatran, $40.2 \%$ received a recommendation-concordant reduced dose, and $59.8 \%$ were prescribed a recommendation-discordant reduced dose. The reasons for recommendation-discordant use in dabigatran included normal renal function (above $\mathrm{CrCl} 50$ 
Table 1. Baseline Characteristics according to Labeled Use

\begin{tabular}{|c|c|c|c|c|}
\hline & On-label standard dose & On-label reduced dose & Off-label underdose & Off-label overdose \\
\hline Number (proportion, \%) & $959(31.1)$ & $949(30.8)$ & $1122(36.4)$ & $50(1.6)$ \\
\hline Dose discrepancy & $284(29.6)$ & $483(50.9)$ & 706 (62.9) & $46(92.0)$ \\
\hline Age (yr) & $65.2 \pm 8.99$ & $73.62 \pm 8.77$ & $72.18 \pm 7.82$ & $72.28 \pm 8.04$ \\
\hline Age $<65$ & $401(41.8)$ & $134(14.1)$ & $165(14.7)$ & $6(12.0)$ \\
\hline Age 65-74 & $423(44.1)$ & $339(35.7)$ & 473 (42.2) & $23(46.0)$ \\
\hline Age $\geq 75$ & $135(14.1)$ & $476(50.2)$ & $484(43.1)$ & $21(42.0)$ \\
\hline Female & $312(32.5)$ & $487(51.3)$ & $521(46.4)$ & $35(70.0)$ \\
\hline Height (cm) & $165.47 \pm 8.12$ & $160.43 \pm 9.48$ & $161.94 \pm 9.11$ & $157.48 \pm 7.55$ \\
\hline Weight (kg) & $70.27 \pm 11.00$ & $61.68 \pm 11.56$ & $64.99 \pm 10.70$ & $58.78 \pm 7.74$ \\
\hline$\leq 60 \mathrm{~kg}$ & $160(16.7)$ & 495 (52.2) & $370(33.0)$ & $35(70.0)$ \\
\hline $\mathrm{BMI}\left(\mathrm{kg} / \mathrm{m}^{2}\right)$ & $25.61 \pm 3.26$ & $23.86 \pm 3.30$ & $24.76 \pm 3.44$ & $23.74 \pm 2.99$ \\
\hline Serum creatinine (mg/dL) & $0.91 \pm 0.20$ & $0.95 \pm 0.29$ & $0.93 \pm 0.27$ & $0.98 \pm 0.31$ \\
\hline $\mathrm{CrCl}(\mathrm{mL} / \mathrm{min})$ & $79.74 \pm 22.54$ & $60.36 \pm 23.43$ & $65.46 \pm 20.52$ & $53.84 \pm 16.52$ \\
\hline $15-30$ & $0(0)$ & $53(5.6)$ & $11(1.0)$ & $1(2.0)$ \\
\hline $30-50$ & $41(4.3)$ & $327(34.5)$ & $214(19.1)$ & $30(60.0)$ \\
\hline$\geq 50$ & 918 (95.7) & $569(60.0)$ & $897(79.9)$ & $19(38.0)$ \\
\hline Heart failure & $138(14.4)$ & $133(14.0)$ & $132(11.8)$ & $4(8.0)$ \\
\hline Hypertension & $696(72.6)$ & $741(78.1)$ & $898(80.0)$ & $38(76.0)$ \\
\hline Diabetes & $300(31.3)$ & $281(29.6)$ & 376 (33.5) & $11(22.0)$ \\
\hline Previous stroke/TIA/TE & $239(24.9)$ & $175(18.4)$ & $229(20.4)$ & $13(26.0)$ \\
\hline Vascular disease & $67(7.0)$ & $69(7.3)$ & $81(7.2)$ & $0(0)$ \\
\hline Previous bleeding & $46(4.8)$ & $92(9.7)$ & $165(14.7)$ & $1(2.0)$ \\
\hline Major bleeding & $13(1.4)$ & $18(1.9)$ & $30(2.7)$ & $1(2.0)$ \\
\hline Intracranial bleeding & $11(1.1)$ & $15(1.6)$ & $20(1.8)$ & $1(2.0)$ \\
\hline $\mathrm{CHA}_{2} \mathrm{DS}_{2}$-VASc score & $2.80 \pm 1.39$ & $3.53 \pm 1.42$ & $3.48 \pm 1.41$ & $3.58 \pm 1.43$ \\
\hline Score $\geq 2$ & $829(86.4)$ & $913(96.2)$ & $1077(96.0)$ & $49(98.0)$ \\
\hline HAS-BLED score & $1.68 \pm 0.92$ & $2.07 \pm 0.85$ & $2.06 \pm 0.82$ & $2.04 \pm 0.81$ \\
\hline Previous warfarin use & $45(4.7)$ & $75(7.9)$ & $36(3.2)$ & $5(10.0)$ \\
\hline \multicolumn{5}{|l|}{ Concomitant medication } \\
\hline Dronedarone & $12(1.3)$ & $24(2.5)$ & $50(4.5)$ & $2(4.0)$ \\
\hline Aspirin & $54(5.6)$ & $77(8.1)$ & $49(4.4)$ & $4(8.0)$ \\
\hline Clopidogrel & $18(1.9)$ & $42(4.4)$ & $72(6.4)$ & $2(4.0)$ \\
\hline Cilostazol & $3(0.3)$ & $7(0.7)$ & $8(0.7)$ & $0(0)$ \\
\hline 1 or more antiplatelet use & $70(7.3)$ & $111(11.7)$ & 124 (11.1) & $4(8.0)$ \\
\hline
\end{tabular}

$\mathrm{BMI}$, body mass index; $\mathrm{CrCl}$, creatinine clearance; $\mathrm{TE}$, thromboembolic events; $\mathrm{TIA}$, transient ischemic attack.

Variables are presented as number (percentage) or mean \pm standard deviation.

$\mathrm{mL} / \mathrm{min}, \mathrm{n}=480)$ and age younger than 75 years $(\mathrm{n}=380)$. We summarized label adherence for each NOAC in Supplementary Fig. 1 (only online). Apixaban showed the smallest number of patients who met the dose reduction criteria $(12.9 \%)$, whereas edoxaban showed the largest number of patients who met the dose reduction criteria (43.4\%). Compared to realworld prescriptions, edoxaban showed a smaller discrepancy between dose label and prescription, compared to rivaroxaban and apixaban (rivaroxaban vs. edoxaban, apixaban vs. edoxaban, both $p<0.001)$.

\section{Risk factors associated with off-label underdosing of NOAC}

The baseline characteristics by NOAC dosing are presented in
Table 1. To identify factors associated with off-label underdosed NOACs, we compared the baseline characteristics of patients prescribed an off-label underdosed NOAC to patients prescribed an on-label standard dose (Supplementary Table 3, only online). Relative to the on-label standard dose group, patients who received off-label underdosed NOACs were significantly older, more likely women, had a lower body weight, had renal impairment and were more likely to have hypertension (all, $p<0.001$ ). Compared to those with on-label standard dose NOACs, the off-label underdosed group showed significantly higher $\mathrm{CHA}_{2} \mathrm{DS}_{2}$-VASc scores (mean $3.48 \pm 1.41$ vs. $2.80 \pm 1.39$ ) and HAS-BLED scores (mean $2.06 \pm 0.82$ vs. $1.68 \pm 0.92$; all, $p<$ 0.001). Previous bleeding was more common in the off-label underdosed group than the on-label standard dose group 


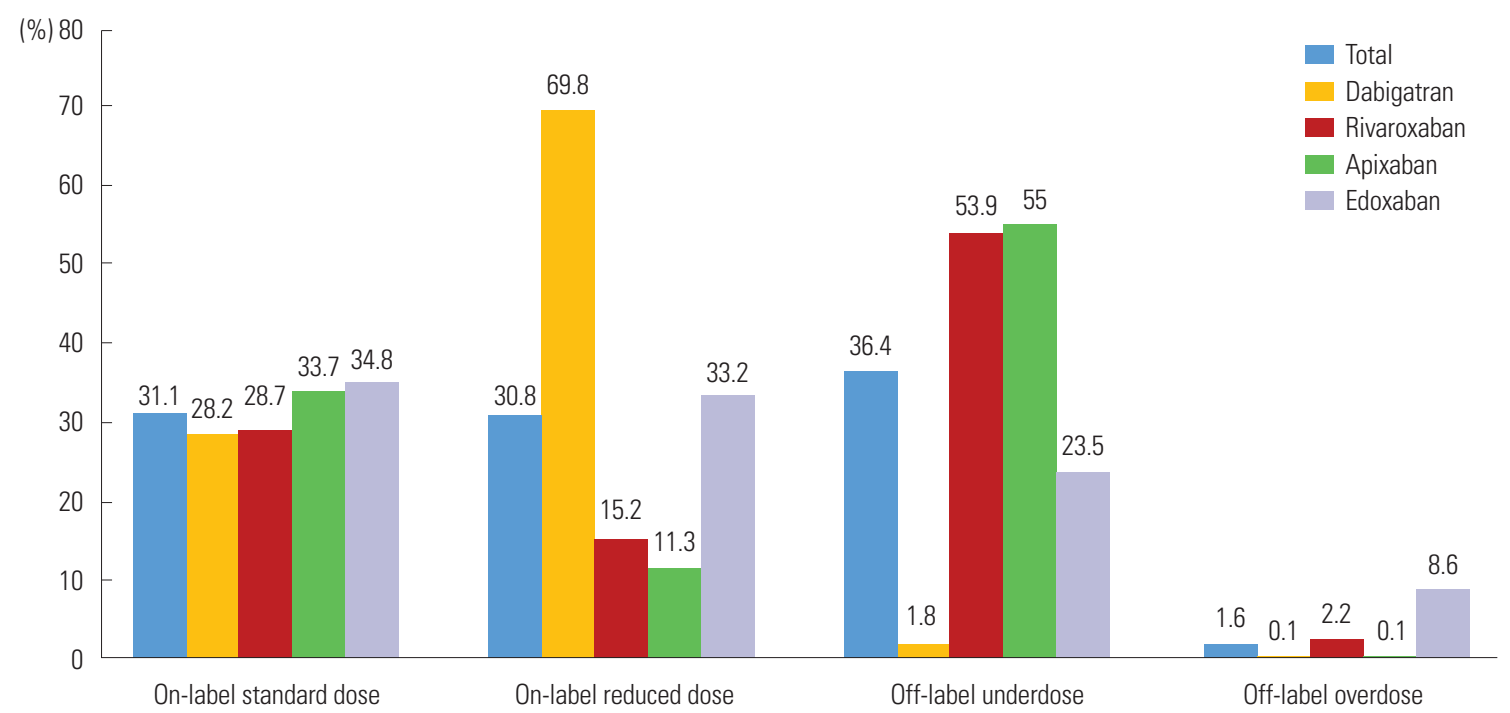

Fig. 1. Differences in drug-specific dose reduction recommendations for each NOAC and discrepancies in NOAC dosing. NOAC, non-vitamin K antagonist oral anticoagulant.
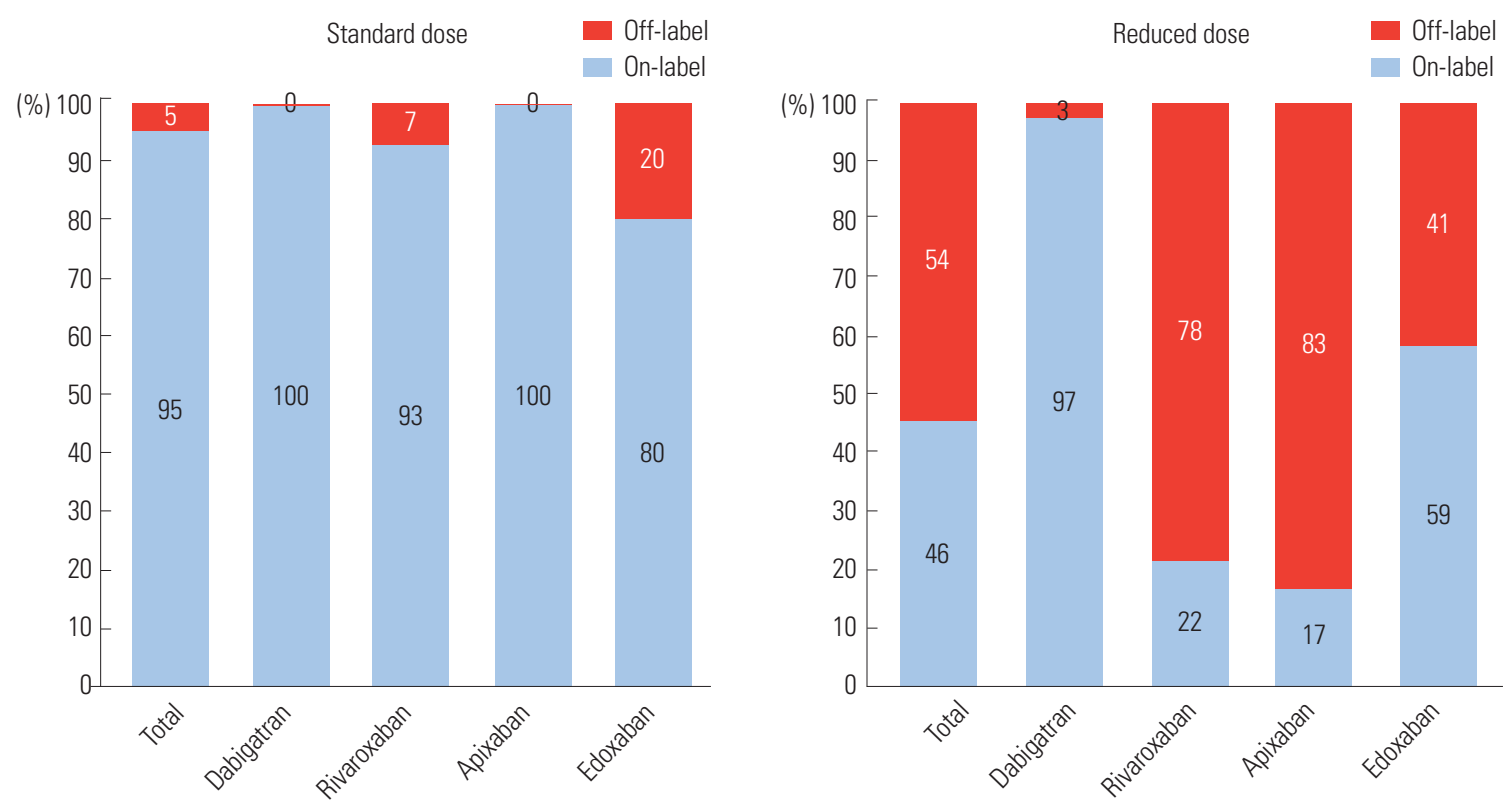

Fig. 2. Label adherence for NOAC dosing in the study population. NOAC, non-vitamin K antagonist oral anticoagulant.

( $14.7 \%$ vs. $4.8 \%, p<0.001$ ), whereas previous thromboembolic events (TE) were more common in the on-label standard dose group compared to the off-label underdosed group ( $24.9 \%$ vs. $20.4 \%, p=0.014)$. Rates of one or more concomitant antiplatelet use were significantly higher in the off-label underdosed group than the on-label standard dose group (11.1\% vs. $7.3 \%$, $p=0.003)$. Dronedarone use was more common in the off-label underdosed group compared to the on-label standard dose group ( $4.5 \%$ vs. $1.3 \%, p<0.001)$. In the multivariable logistic regression, old age ( $\geq 75$ years), women, a lower body weight $(\leq 60$ $\mathrm{kg}$ ), renal impairment ( $\mathrm{CrCl} \leq 50 \mathrm{~mL} / \mathrm{min})$, prevalent hypertension, previous stroke/transient ischemic attack (TIA)/TE, previous bleeding, dronedarone use, and anti-platelet use were associated with off-label underdosing (Fig. 3). These findings were similar when the $\mathrm{CHA}_{2} \mathrm{DS}_{2}$-VASc and HASBLED scores were included in multivariable analysis (Supplementary Table 4 , only online).

Each NOAC has different dose reduction criteria and the proportions of off-label underdosed prescription were different among NOACs. We evaluated the associated factors with off-label underdosed prescription in each NOAC group (Supplementary Tables 5-8, Supplementary Fig. 2, only online). Although the factors varied across the types of NOACs due to patient numbers of each group, the associated factors with offlabel underdosing were largely consistent with the results from total study population (Fig. 3). 


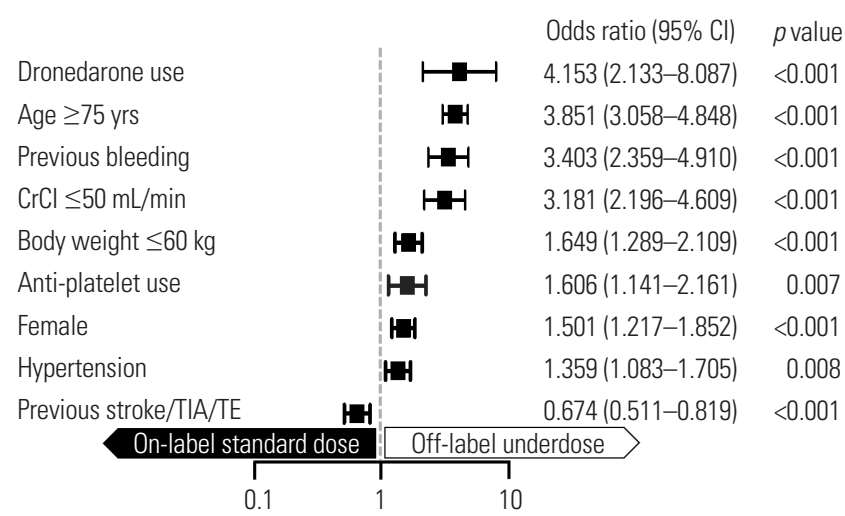

Fig. 3. Factors associated with off-label underdosing. $\mathrm{CrCl}$, creatinine clearance; TE, thromboembolic events; TIA, transient ischemic attack; $\mathrm{Cl}$, confidence interval.

\section{DISCUSSION}

\section{Main findings}

In this study, we analyzed label adherence for dosing across four NOACs and factors associated with off-label underdosing in patients with $\mathrm{AF}$ in routine clinical practice. In this large-scale Asian AF cohort who were prescribed NOACs, the main findings were as follows: 1) off-label NOAC prescription was frequently observed, and label adherence for NOAC dosing was about $60 \%$; 2) most standard doses of NOAC were prescribed as in label, although more than half of the reduced doses of NOAC were prescribed off-label; 3 ) edoxaban showed the highest dose recommendation adherence, whereas rivaroxaban showed the lowest dose recommendation adherence; and 4 ) old age ( $\geq 75$ years), women, lower body weight $(\leq 60$ $\mathrm{kg}$ ), renal impairment $(\mathrm{CrCl} \leq 50 \mathrm{~mL} / \mathrm{min})$, prevalent hypertension, previous stroke/TIA/TE, previous bleeding, dronedarone use, and anti-platelet use were associated with off-label underdosing prescriptions of NOACs. These findings could give important insights into real-world NOAC prescription behavior in Asian populations.

\section{Dose reduction criteria for individual NOACs and their complexity}

Patients taking NOACs have favorable risk-benefit profiles, few drug interactions, and require less intensive monitoring than those taking warfarin. Therefore, NOACs are being prescribed more frequently for stroke prevention in nonvalvular AF. ${ }^{2-4,17}$ Each NOAC requires a different dose reduction strategy as described in their phase 3 clinical trials (Supplementary Table 1, only online). The proportion of patients who indicated dose reductions were different among pivotal clinical trials (Supplementary Fig. 3, only online).$^{5-8}$ Dabigatran did not have dose reduction criteria in RE-LY, and patients were randomly assigned doses of $150 \mathrm{mg}$ or $110 \mathrm{mg}$ twice daily. ${ }^{5}$ In the ROCKET AF trials, $21 \%$ of the rivaroxaban group who had moderate renal impairment $(\mathrm{CrCl} 30-49 \mathrm{~mL} / \mathrm{min})$ received rivaroxaban 15 mg once daily. ${ }^{6}$ In the ARISTOTLE trials, less than $5 \%$ of the study population was recommended for dose reduction. ${ }^{7}$ In the high-dose regimen edoxaban group in the ENGAGE AFTIMI 48 trial, $25 \%$ of the patients indicated a dose reduction. ${ }^{8}$ We applied dose reduction criteria for each NOAC to the total population to calculate the proportion of patients who were indicated for a dose reduction (Supplementary Fig. 4, only online). Apixaban permitted the smallest proportion of the population (9.6\%) to use a reduced dose by their drug label, whereas edoxaban permitted the largest proportion of the population (44\%). The proportion of indicated dose reductions was quite similar in cases of rivaroxaban, compared to clinical trials (22\% vs. $21 \%$ ). For apixaban and edoxaban, more patients were indicated to have a reduced dose of NOACs in the real-world population, compared with clinical trials (apixaban, $9.6 \%$ vs. $4.7 \%$; and edoxaban $44 \%$ vs. $25.4 \%$ ). These findings might be caused by a lower body weight in Asian populations. Interestingly, the agreement of dose reductions among all four NOAC drug labels was observed only in $5.6 \%$ of the total study population. Forty-three percent of the total population was recommended a standard dose of NOAC. However, in $49.3 \%$ of patients, a discrepancy in NOAC dosing was observed. Therefore, half of patients might be prescribed either a standard dose or reduced dose with different NOACs, not violating the dose reduction label.

\section{Label adherence of NOAC dosing}

The appropriate use of NOACs is an emerging issue. There are several reports regarding the labeled use of NOAC in patients with AF. Recently, in the ORBIT-AF II (Outcomes Registry for Better Informed Treatment of Atrial Fibrillation II) registry, $12.8 \%$ of the total study population received NOACs inconsistent with the drug label (9.4\% with an off-label underdose and $3.4 \%$ with an off-label overdose).${ }^{10}$ In a large recent U.S. claims database, $83.7 \%$ of the total patients were prescribed the recommended dose of NOACs, while $12.0 \%$ and $4.3 \%$ of the study patients received off-label underdose and overdose NOACs, respectively. ${ }^{11}$ Although reduced dose NOACs are commonly used in Asian patients with AF, there are no data about "label adhered dosing" in the Asian population. According to Taiwanese nationwide data, almost $90 \%$ of the total study population was prescribed a reduced dose of NOACs (110 mg twice daily dabigatran and $10 \mathrm{mg}$ to $15 \mathrm{mg}$ once daily rivaroxaban). ${ }^{12}$ Additionally, in the Korean nationwide data, $40-60 \%$ of the patients were prescribed a reduced dose of NOACs. ${ }^{14}$ However, neither studies reported label adherence for NOAC dosing. In our study, $67.3 \%$ of patients were prescribed a reduced dose of NOACs, regardless of label adherence, and $38 \%$ of the total population did not meet the recommended drug labeling use. Most off-label dosing was considered as off-label underdose NOAC use (96\% of off-label dosing population). Namely, patients who were a recommended standard dose of NOACs, based on their baseline characteristics, frequently received a reduced dose of NOACs. 
There are several explanations regarding the reason for the preference of reduced doses of NOAC prescriptions in the Asian population. First, the Asian population has a lower body mass, and some clinicians who targeted a low international normalized ratio in older adult patients replaced warfarin with a reduced dose of NOAC. ${ }^{12}$ Furthermore, different approved doses were used in some Asian countries based on clinical evidence. In Japan, $15 \mathrm{mg}$ rivaroxaban was recommended as a standard dose instead of $20 \mathrm{mg}$, based on the J-ROCKET-AF study. ${ }^{18}$ Second, the Asian population tends to have a higher risk of intracranial hemorrhage (ICH) than the Western population, for which clinicians feel it important not to increase the risk of bleeding. ${ }^{13}$ Based on these results, we could assume that physicians tend to choose reduced dose NOACs for patients regarded as more fragile to bleeding. In addition, when patients are in the "borderline gray zone" by label, physicians were more likely to select a reduced dose NOAC. Compared to the on-label standard dose group, patients with an off-label underdose of NOACs were more likely to have a dosing discrepancy based on the drug-specific label (62.9\% vs. $29.6 \%$, respectively; $p<$ $0.001)$.

\section{Clinical implications of label adherence for NOAC dosing}

A few previous reports have outlined the clinical consequences of label adherence. In the ENGAGE AF-TIMI 48 trial, a lowdose edoxaban regimen posed a significantly higher ischemic stroke risk, whereas patients with the same dose of edoxaban who met one of the dose reduction criteria showed a comparable risk of stroke to those taking warfarin. ${ }^{8}$ Recently, adverse clinical consequences for off-label dosing have been reported from real-world data. In the ORBIT-AF II registry, compared to the recommended dose of NOACs, off-label doses of NOACs showed higher adverse events rates. ${ }^{10}$ Particularly, an offlabel underdose of NOAC was associated with a worsened effectiveness with no benefit in safety. ${ }^{10,11}$ Notably, the estimated stroke risk was significantly higher in the off-label underdose group, compared to the on-label standard dose group. To improve the clinical outcomes of Asian AF patients, an increase in label adherence for NOAC dosing might be needed.

\section{Consideration of optimal NOAC doses for Asian AF patients}

There are a few reports suggesting that lower dose NOACs regarded as "off-label underdose" by current labels could be safe and effective in the Asian population. Although $15 \mathrm{mg}$ rivaroxaban was recommended as a standard dose in patients without renal impairment in the J-ROCEKT-AF trial, the number of patients was small to generalize for Asian patients. ${ }^{18}$ However, although dabigatran showed different positions in terms of label adherence, recently, patients prescribed recommendation-discordant $110 \mathrm{mg}$ dabigatran present comparable results in effectiveness and safety, compared to patients with
150 mg dabigatran, among Korean AF patients. ${ }^{16}$ Based on the Taiwanese nationwide cohort, universally-prescribed reduced doses of dabigatran and rivaroxaban, regardless of label adherence, showed better effectiveness and safety, compared to the real-world warfarin group. ${ }^{12}$ Considering that Asians have a lower body mass, more prevalent ICH, and generally a lower time in the therapeutic range, further investigation is needed to assess the optimal doses of NOACs and net clinical effects of off-label NOAC prescription in the Asian AF population. ${ }^{12,13,19,20}$

\section{Study limitations}

There are several limitations in the present study. We analyzed NOAC dosing patterns based on patients and their baseline characteristics. However, body weight, renal function, and concomitant drug use could be modified during follow-up and might affect the physician's choice of NOAC dosing. In addition, in this study, we did not include follow-up data; therefore, clinical outcomes according to inappropriate NOAC dosing were not evaluated. Further investigation will be needed to determine the clinical implications of NOAC dosing in the Korean AF population. Lastly, this study analyzed the multicenter prospective registry of Korean population. Thus, ethnic uniformity should be considered, and the generalization of the study results should be proceeded with caution. Moreover, among East Asian countries, definitions of "on-label dose" for rivaroxaban differ, as do proportions of low dose prescription of NOACs. Despite these limitations, this study describes a prospectively-collected large number of Korean AF patients. Our findings reflect the real-world clinical practice patterns of NOAC dosing in contemporary Asian patients with AF.

In conclusion, in real-world practice, half of Korea patients treated with NOACs received off-label reduced dose. Older age, female sex, lower body weight, impaired renal function, previous stroke or bleeding history, hypertension, and concomitant dronedarone or antiplatelet agent use were independently associated with off-label underdose prescription. Further study is needed to investigate the clinical outcomes of off-label prescription of NOACs, especially in Asians.

\section{ACKNOWLEDGEMENTS}

This study was supported by the Korea National Research Foundation of Korea (NRF) funded by the Ministry of Education, Science and Technology (2014R1A1A2A16055218), Korea Government (Ministry of Science, ICT and Future Planning) (NRF-2015R1C1A2A01054767), the Korean Healthcare Technology R\&D project funded by the Ministry of Health and Welfare (HI15C1200), and also by the Technology Innovation Program or Industrial Strategic Technology Development Program (10052668, development of wearable self-powered energy source and low-power wireless communication system for a pacemaker) funded by the Ministry of Trade, Industry and Energy (MOTIE, Sejong, Korea). 


\section{AUTHOR CONTRIBUTIONS}

Conceptualization: Choi EK. Data curation: Joung B, Lee SR. Formal analysis: Lee SR, Park JS. Funding acquisition: Joung B, Choi EK. Investigation: Lee SR, Cha MJ. Methodology: Kim TH, Park J, Park JK. Project administration: Lee JM, Kang KW, Shim J. Resources: Uhm JS, Kim J. Software: Kim C, Kim JB. Supervision: Park HW. Validation: Park JS. Visualization: Lee SR. Writing_original draft: Lee SR. Writing_review \& editing: Joung B, Choi EK, Lee Y.

\section{ORCID iDs}

So-Ryoung Lee Young Soo Lee Ji-Suck Park Myung-Jin Cha Tae-Hoon Kim Junbeom Park Jin-Kyu Park Jung-Myung Lee Ki-Woon Kang Jaemin Shim Jae-Sun Uhm Jun Kim Changsoo Kim Jin-Bae Kim Hyung Wook Park Boyoung Joung Eue-Keun Choi https://orcid.org/0000-0002-6351-5015 https://orcid.org/0000-0002-8229-8300 https://orcid.org/0000-0001-6924-7106 https://orcid.org/0000-0001-6180-0157 https://orcid.org/0000-0003-4200-3456 https://orcid.org/0000-0003-2192-9401 https://orcid.org/0000-0001-7931-777X https://orcid.org/0000-0002-1904-5335 https://orcid.org/0000-0002-1361-0022 https://orcid.org/0000-0001-8251-1522 https://orcid.org/0000-0002-1611-8172 https://orcid.org/0000-0002-3573-638X https://orcid.org/0000-0002-5940-5649 https://orcid.org/0000-0002-0293-0901 https://orcid.org/0000-0002-9630-0467 https://orcid.org/0000-0001-9036-7225 https://orcid.org/0000-0002-0411-6372

\section{REFERENCES}

1. Ogilvie IM, Newton N, Welner SA, Cowell W, Lip GY. Underuse of oral anticoagulants in atrial fibrillation: a systematic review. Am J Med 2010;123:638-45.

2. Huisman MV, Rothman KJ, Paquette M, Teutsch C, Diener HC, Dubner SJ, et al. The changing landscape for stroke prevention in AF: findings from the GLORIA-AF registry phase 2. J Am Coll Cardiol 2017;69:777-85.

3. Camm AJ, Accetta G, Ambrosio G, Atar D, Bassand JP, Berge E, et al. Evolving antithrombotic treatment patterns for patients with newly diagnosed atrial fibrillation. Heart 2017;103:307-14.

4. Lee SR, Choi EK, Han KD, Cha MJ, Oh S, Lip GYH. Temporal trends of antithrombotic therapy for stroke prevention in Korean patients with non-valvular atrial fibrillation in the era of non-vitamin K antagonist oral anticoagulants: a nationwide populationbased study. PLoS One 2017;12:e189495.

5. Connolly SJ, Ezekowitz MD, Yusuf S, Eikelboom J, Oldgren J, Parekh A, et al. Dabigatran versus warfarin in patients with atrial fibrillation. N Engl J Med 2009;361:1139-51.

6. Patel MR, Mahaffey KW, Garg J, Pan G, Singer DE, Hacke W, et al.
Rivaroxaban versus warfarin in nonvalvular atrial fibrillation. N Engl J Med 2011;365:883-91.

7. Granger CB, Alexander JH, McMurray JJ, Lopes RD, Hylek EM, Hanna M, et al. Apixaban versus warfarin in patients with atrial fibrillation. N Engl J Med 2011;365:981-92.

8. Giugliano RP, Ruff CT, Braunwald E, Murphy SA, Wiviott SD, Halperin JL, et al. Edoxaban versus warfarin in patients with atrial fibrillation. N Engl J Med 2013;369:2093-104.

9. Kirchhof P, Benussi S, Kotecha D, Ahlsson A, Atar D, Casadei B, et al. 2016 ESC Guidelines for the management of atrial fibrillation developed in collaboration with EACTS. Eur Heart J 2016;37: 2893-962.

10. Steinberg BA, Shrader P, Thomas L, Ansell J, Fonarow GC, Gersh BJ, et al. Off-label dosing of non-vitamin $\mathrm{K}$ antagonist oral anticoagulants and adverse outcomes: the ORBIT-AF II Registry. J Am Coll Cardiol 2016;68:2597-604.

11. Yao X, Shah ND, Sangaralingham LR, Gersh BJ, Noseworthy PA. Non-vitamin $\mathrm{K}$ antagonist oral anticoagulant dosing in patients with atrial fibrillation and renal dysfunction. J Am Coll Cardiol 2017;69:2779-90.

12. Chan YH, Kuo CT, Yeh YH, Chang SH, Wu LS, Lee HF, et al. Thromboembolic, bleeding, and mortality risks of rivaroxaban and dabigatran in Asians with nonvalvular atrial fibrillation. J Am Coll Cardiol 2016;68:1389-401.

13. Shen AY, Yao JF, Brar SS, Jorgensen MB, Chen W. Racial/ethnic differences in the risk of intracranial hemorrhage among patients with atrial fibrillation. J Am Coll Cardiol 2007;50:309-15.

14. Cha MJ, Choi EK, Han KD, Lee SR, Lim WH, Oh S, et al. Effectiveness and safety of non-vitamin $\mathrm{K}$ antagonist oral anticoagulants in Asian patients with atrial fibrillation. Stroke 2017;48:3040-8.

15. Kim H, Kim TH, Cha MJ, Lee JM, Park J, Park JK, et al. A prospective survey of atrial fibrillation management for real-world guideline adherence: COmparison study of Drugs for symptom control and complication prEvention of Atrial Fibrillation (CODE-AF) registry. Korean Circ J 2017;47:877-87.

16. Lee KH, Park HW, Lee N, Hyun DY, Won J, Oh SS, et al. Optimal dose of dabigatran for the prevention of thromboembolism with minimal bleeding risk in Korean patients with atrial fibrillation. Europace 2017;19(suppl_4):iv1-9.

17. Ruff CT, Giugliano RP, Braunwald E, Hoffman EB, Deenadayalu N, Ezekowitz MD, et al. Comparison of the efficacy and safety of new oral anticoagulants with warfarin in patients with atrial fibrillation: a meta-analysis of randomised trials. Lancet 2014;383:955-62.

18. Hori M, Matsumoto M, Tanahashi N, Momomura S, Uchiyama S, Goto S, et al. Rivaroxaban vs. warfarin in Japanese patients with atrial fibrillation - the J-ROCKET AF study. Circ J 2012;76:2104-11.

19. Oh S, Goto S, Accetta G, Angchaisuksiri P, Camm AJ, Cools F, et al. Vitamin $\mathrm{K}$ antagonist control in patients with atrial fibrillation in Asia compared with other regions of the world: real-world data from the GARFIELD-AF registry. Int J Cardiol 2016;223:543-7.

20. Chiang CE, Wang KL, Lip GY. Stroke prevention in atrial fibrillation: an Asian perspective. Thromb Haemost 2014;111:789-97. 